\title{
Metodología para la evaluación de la condición de cambiadores de derivaciones bajo carga según su índice de salud
}

\section{A methodology for assess the on-load tap changers condition according to their health index}

Edgar Herney Cañar-Ramirez¹, Guillermo Aponte-Mayor², Andrés Felipe Cerón-Piamba ${ }^{3}$

Cañar-Ramirez, E.H; Aponte-Mayor, G; Cerón-Piamba, A.F. Metodología para la evaluación de la condición de cambiadores de derivaciones bajo carga según su índice de salud. Tecnología en Marcha. Vol. 34, especial. ALTAE. Diciembre 2021. Pág 126-141.

doi) https://doi.org/10.18845/tm.v34i7.6020

1 Celsia Colombia S.A. Colombia.

2 Universidad del Valle. Colombia. 


\title{
Palabras clave
}

Cambiador de derivaciones bajo carga (OLTC); metodología; mantenimiento; gestión de activos; índice de salud; condición; clasificación; criterios de selección.

\section{Resumen}

En este trabajo se presenta una metodología para evaluar el cambiador de derivaciones bajo carga OLTC según su índice de salud, construida a partir del principio de funcionamiento y las características generales de este equipo. Dado que las intervenciones en estos equipos tradicionalmente han dependido de los historiales de mantenimiento y de fabricantes, con esta metodología se busca migrar al mantenimiento basado en la condición del equipo, optimizando las inversiones financieras y de personal, generando mayor confiabilidad en el sistema y obteniendo una mejor gestión de los activos. Para ello, inicialmente se identificaron los modos de falla que pueden presentarse, seleccionando así las pruebas que más aportan para un diagnóstico del OLTC, posteriormente se realizó el desarrollo de la metodología combinando de manera ponderada las pruebas y finalmente se integró esta metodología a la herramienta de estimación del índice de Salud de transformadores desarrollada por el Grupo de investigación en alta tensión de la Universidad del Valle GRALTA. La metodología desarrollada se validó con un caso de estudio.

\section{Keywords}

On-load tap changer (OLTC); methodology; maintenance; asset management; health index; condition; classification; selection criteria.

\begin{abstract}
This work presents a methodology to evaluate the OLTC on-load tap changer according to its health index, built from the operating principle and the general characteristics of this equipment. Given that interventions in this equipment have traditionally depended on maintenance and manufacturer records, this methodology seeks to migrate to maintenance based on the condition of the equipment, optimizing financial and personnel investments, generating greater reliability in the system and obtaining better asset management. To do this, initially the failure modes that may occur were identified, thus selecting the tests that most contribute to a diagnosis of OLTC, later the development of the methodology was carried out combining the tests in a weighted way and finally this methodology was integrated into the tool of estimation of the Transformer Health index developed by the High Voltage Research Group of the Universidad del Valle GRALTA. The methodology developed was validated with a case study.
\end{abstract}

\section{Introducción}

Los transformadores son parte fundamental en los sistemas eléctricos de potencia (SEP), ya que son los encargados de transformar la energía eléctrica en diferentes niveles de tensión y corriente, logrando así que se reduzcan las pérdidas en la transmisión, desde los centros de generación hasta los centros de consumo. Existen más de 100.000 transformadores de potencia operando en Estados Unidos y alrededor de 400.000 en todo el mundo, de los cuales en Colombia se estima que hay aproximadamente 1000 en funcionamiento [1]. Dado que el costo de estos equipos puede llegar a representar hasta el 60\% del total de inversión de una 
subestación y que rara vez se dispone de un equipo de reserva para una contingencia, resulta transcendental conservar las condiciones normales de trabajo extendiendo así su vida útil, la cual está estimada en 40 años [1][2].

Según IEEE C57.140, el transformador se encuentra dividido en 9 sistemas: devanados, núcleo, pasatapas, tanque de enfriamiento, sistema conservador de aceite, refrigerador, cambiador de derivaciones bajo carga (OLTC), cambiador de derivaciones sin carga y tanque del transformador [3]oil reclamation, testing methods for the determination of remaining insulation (paper[1]. La gestión para el mantenimiento debe considerar todos los sistemas del transformador, incluyendo el cambiador de derivaciones, encargado de la regulación y control de los niveles de tensión y Su consideración es importante; ya que estudios realizados indican que por problemas en los OLTC, se generan entre el $20 \%$ y $40 \%$ de las fallas del transformador [1][4][5]Hydro-Québec's Research Institute (IREQ[6].

El Grupo de Investigación en Alta Tensión (GRALTA) de la Universidad del Valle, ha trabajado en estimación del IS del transformador, pero sin los OLTC de manera específica. Con este trabajo se propone una metodología para la evaluación de la condición del OLTC según su índice de salud, que puede integrarse a la herramienta de estimación del IS del transformador desarrollada por el grupo de investigación.

\section{Antecedentes}

Índice de salud como herramienta de diagnóstico de transformadores de potencia

El índice de salud es una herramienta que ha sido empleada a través del tiempo para conocer la condición de operación de diversos activos, en la cual principalmente se emplean técnicas combinatorias con reglas de lógica difusa o combinaciones lineales, para poder obtener un indicador de la condición de un equipo.

Anteriormente, las empresas basaban sus planes de mantenimiento preventivo en los números de operaciones, historiales de mantenimiento y cumplimiento de la vida útil de estos equipos, pero en la actualidad se prefieren técnicas que permitan tomar decisiones con base en la condición real de los equipos.

Las metodologías basadas en la condición permitan la integración de la mayor cantidad de pruebas, información cronológica, información técnica como número de operaciones, siendo esta una de las más eficientes desde una visión técnico-económica, ya que permite dar prioridad a los equipos que necesitan de la intervención de manera más oportuna y segura [1] [4][5]Hydro-Québec's Research Institute (IREQ.

Los transformadores de potencia de alto voltaje están sujetos a diferentes tipos de mecanismos de degradación [7].
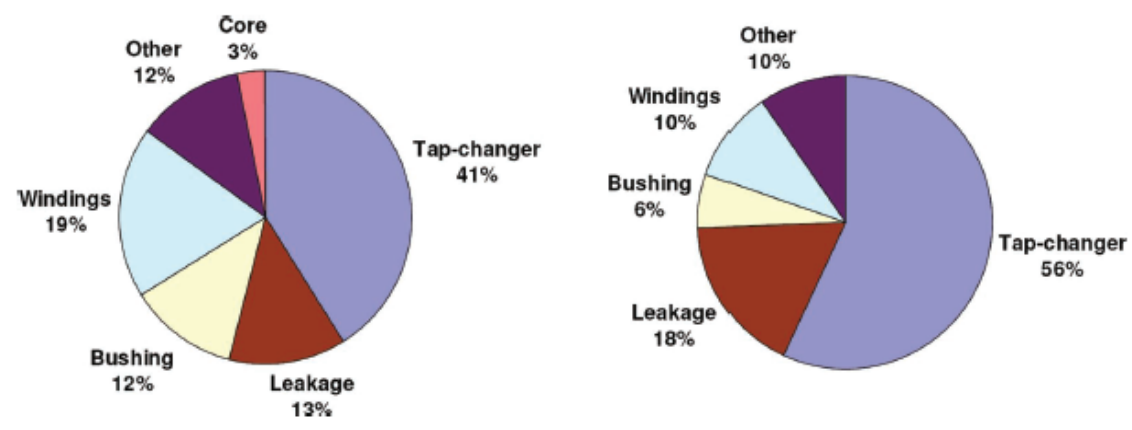

Figura 1. Repartición de fallas en el transformador de potencia. Fuente. Adaptado de [7]. 
Razones por las cuales es importante considerar al OLTC para obtener un índice de salud del transformador de potencia.

En la figura 2 se muestra el esquema de la herramienta para estimar el IS.

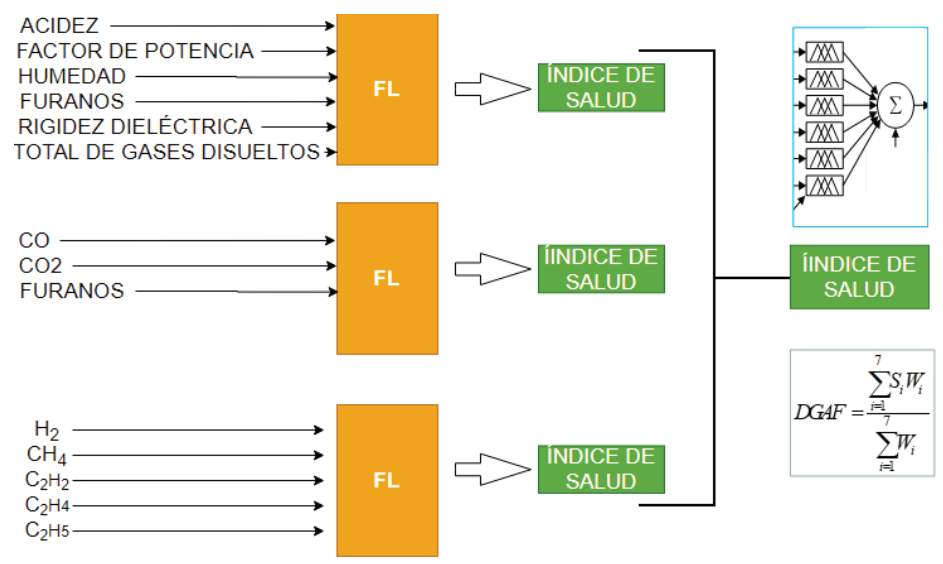

Figura 2. Obtención del índice de salud del transformador de potencia con la herramienta GRALTA.Fuente. Adaptado de [4].

Con el fin de lograr integrar la condición del OLTC al modelo presentado en la figura 2 se debe adicionar un módulo que evalué las pruebas pertinentes para obtener un índice de salud del OLTC y así poderlo considerar al calcular la condición general del transformador, como se muestra en la figura 3.

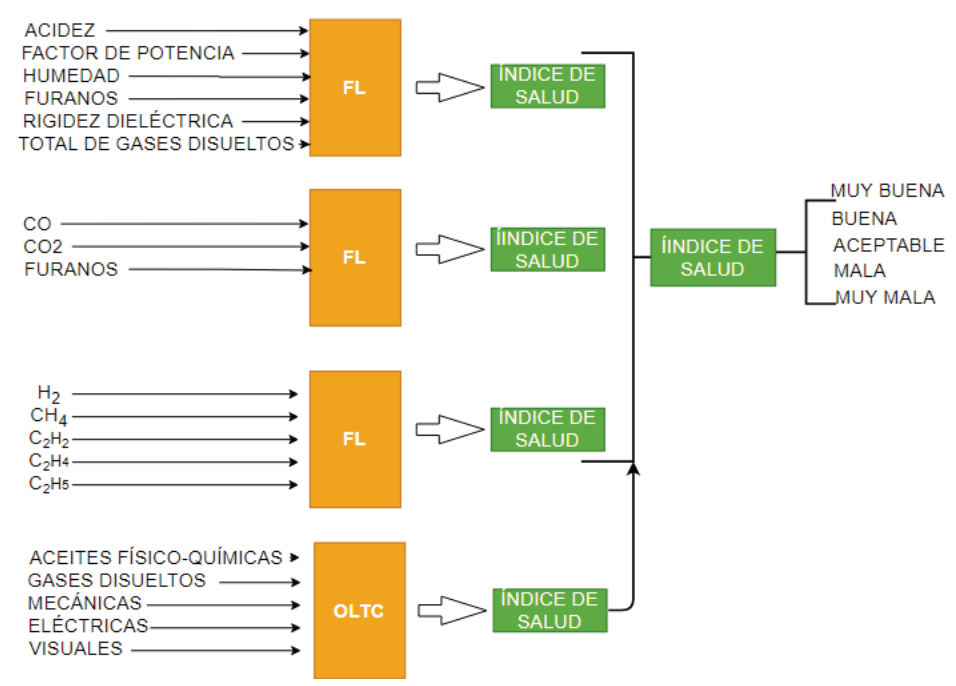

Figura 3. Adición del módulo del OLTC a las metodologías existentes.

\section{Generalidades de los OLTC}

El (OLTC) es una parte importante del transformador de potencia, y sus condiciones de funcionamiento están directamente relacionadas con la estabilidad y la seguridad del transformador y el (SEP). El OLTC es uno de los componentes del transformador con la tasa de falla más alta [4][5]Hydro-Québec's Research Institute (IREQ. Según las estadísticas, las fallas en el OLTC son básicamente mecánicas, térmicas y eléctricas, como contactos flojos los cuales desencadenan en altas temperaturas y arcos eléctricos que deterioran la calidad del medio 
dieléctrico, mecanismos atascados, engranajes deslizantes y acción de rechazo [1][4][7][8]. En los últimos años, el análisis de gases disueltos en aceite y otras pruebas de líquido aislante se han utilizado como herramientas efectivas para detectar problemas en los OLTC. Se han desarrollado pautas y algoritmos específicos para evaluar los resultados del OLTC normales y anormales contribuyendo en la evaluación de la condición [1][9][10] [11][12][13].

\section{Tipos de cambiadores de derivaciones bajo carga}

Existen tres tipos principales de OLTC, estos son:

1. OLTC de tipo Resistivos con contactos de arco en aceite, normalmente son conectados en el lado de alta tensión, operan entre 5 milisegundos después de que da la orden de cambio de toma, comparte la cuba del transformador ver figura 4, cuentan con una resistencia de transición de alta potencia, usada para que no quede flotando el devanado principal en el cambio de toma.

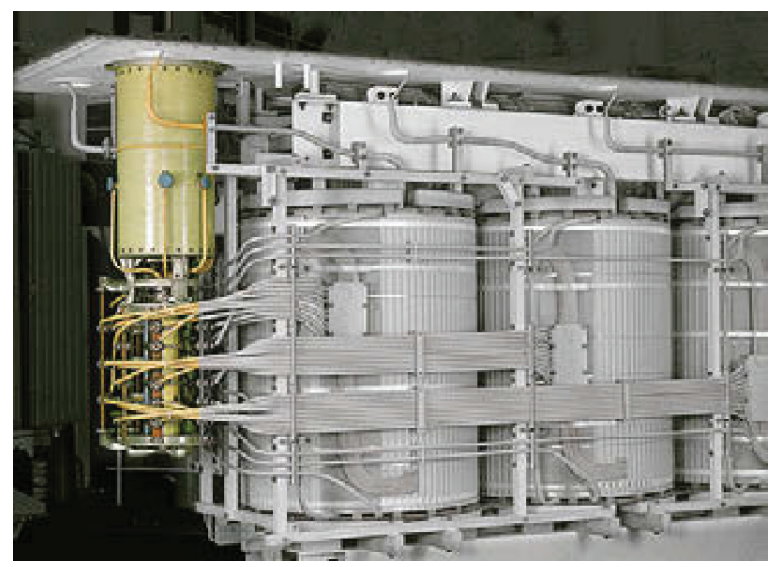

Figura 4. Disposición física del OLTC de tipo resistivo. Fuente. Adaptado de [14].

2. OLTC de tipo Reactivos con contactos de arco en aceite, conectados por el lado de baja tensión, no disipan tanta energía como las resistencias, diseñados para soportar la totalidad de la corriente de carga y la corriente circulante por largos periodos de tiempo, opera en una posición de puente entre terminales, mejorando la regulación, haciéndola más fina y duplicando las posiciones de servicio[15], tiene un compartimiento aparte del transformador ver figura 5.
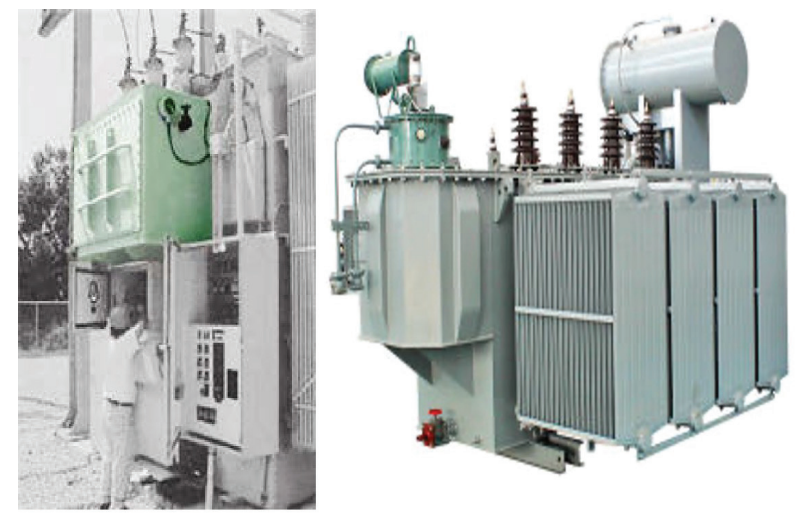

Figura 5. Disposición física del OLTC de tipo reactivo. Fuente. Adaptado de [15].

3. Contactos de arco en una botella de vacío, con el paso del tiempo, la tecnología de conmutación de vacío se ha convertido en la tecnología predominante en subestaciones de media tensión y ha reemplazado la tecnología de aceite. Su superioridad sobre las 
tecnologías de conmutación en los rangos de potencia baja y media se basa en una serie de características técnicas como la eliminación de subproductos de carbono, características de conmutación constantes o incluso mejoradas a lo largo de su vida útil, la alta tasa de condensación de vapor metálico prolongando la vida útil del contacto, baja resistencia de contacto, garantiza tiempos de arco cortos máximo un medio ciclo, [16] [17].

Pruebas, métodos de identificación de falla y programación de mantenimiento.

\section{Pruebas}

El uso de pruebas es cada vez más importante, ya que proporcionan datos para diagnosticar la condición de los aparatos eléctricos. Esta información se puede utilizar para detectar fallas incipientes, proporcionar una indicación de su gravedad e identifican tendencias de envejecimiento a largo plazo. En el entorno actual, donde las pruebas fuera de servicio de los aparatos no siempre son posibles, poder adquirir información en servicio, es una ventaja [17]. Las pruebas actualmente utilizadas se describen a continuación:

- Gases disueltos en el aceite (DGA).

- Comprobaciones de continuidad.

- Mediciones estáticas.

- Resistencias dinámicas.

- Corriente dinámica.

- Temperatura.

- Contenido de agua.

- Tensión de ruptura dieléctrica.

- Número de neutralización.

- Recuento de partículas y análisis cualitativo.

- Los metales totales en el aceite.

- Análisis vibroacústico.

- Desgaste de los contactos.

- Inspecciones visuales.

Métodos de identificación de falla y programación de mantenimiento

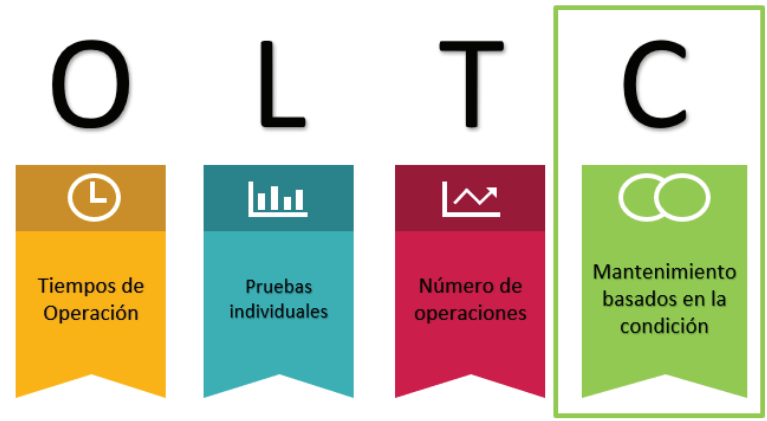

Figura 6. Métodos de identificación de fallas y programación de mantenimiento. 


\section{Metodologías para la evaluación de la condición de cambiadores de derivaciones bajo carga y criterios de selección}

Existen diferentes metodologías que permiten conocer la condición de un equipo y estas se basan en pruebas técnicas, inspecciones visuales y condiciones de funcionamiento, pero cada una se diferencia por los métodos que se utilizan, número de pruebas e importancia que tienen las pruebas en el método. A continuación, se presentan las más conocidas para OLTC.

Cuadro 1. Metodologías utilizadas para evaluar los transformadores de potencia incluyendo el OLTC.

\begin{tabular}{|c|c|c|c|c|}
\hline \multicolumn{5}{|c|}{ Metodologías para la evaluación de la condición del transformador de potencia basadas en indice de salud HI } \\
\hline ITEM & AÑO DE PUBLCACIÓN & AUTOR & MÉTODO DE CÁLCULO & DIFERENCIAS \\
\hline 1 & 2008 & Ali Naderian Jaromi & $H I=60 \% * \frac{\sum_{j=1}^{17} K_{j} H I F_{j}}{\sum_{j=1}^{17} 4 K_{j}}+40 \% * \frac{\sum_{j=18}^{20} K_{j} H I F_{j}}{\sum_{j=18}^{20} 4 K_{j}}$ & $\begin{array}{l}\text { Usa } 20 \text { pruebas de las cuales } 3 \\
\text { son del OLTC }\end{array}$ \\
\hline 2 & 2009 & Ali Naderian Jaromi & $H I=60 \% * \frac{\sum_{j=1}^{21} K_{j} H I F_{j}}{\sum_{j=1}^{21} 4 K_{j}}+40 \% * \frac{\sum_{j=22}^{24} K_{j} H I F_{j}}{\sum_{j=22}^{24} 4 K_{j}}$ & $\begin{array}{c}\text { Usa } 24 \text { pruebas de las cuales } 3 \\
\text { son del OLTC, cambian los pesos } \\
\text { asignados a cada prueba, } \\
\text { también cambian pruebas }\end{array}$ \\
\hline 3 & 2012 & $\begin{array}{l}\text { G. Tanasescu, O. } \\
\text { Dragomir }\end{array}$ & $\begin{array}{l}H I=A_{1} * \frac{\sum_{i=1}^{n-3} C_{i} D I_{i}}{\sum_{i=1}^{n-3} 4 C_{i}}+A_{2} * \frac{\sum_{i=-3}^{n} C_{i} D I_{i}}{\sum_{i=n-3}^{n} 4 C_{i}} \\
R L=E A(P=50 \%, \text { criterio de fin de vida })-E A(t)\end{array}$ & \begin{tabular}{|l} 
Se establece el método general \\
y la formulación matemática \\
indica que solo serán 3 pruebas \\
para el OLTC, las pruebas pueden \\
ser seleccionadas por la \\
metodología, además se trabaja \\
con la vida útil restante
\end{tabular} \\
\hline 4 & 2012 & Juthathip Haema & $\% H I=\left(0.6 * \frac{\sum_{j=1}^{17}\left(K_{j} * H I F_{j}\right)}{\sum_{j=1}^{17} H I F_{j \max } * K_{j}}+0.4 * \frac{\sum_{j=18}^{21}\left(K_{j} * H I F_{j}\right)}{\sum_{j=18}^{21} 4 * K_{j}}\right) * 100$ & $\begin{array}{c}\text { Usa } 21 \text { pruebas de las cuales } 4 \\
\text { son del OLTC, cambian los pesos } \\
\text { asignados a cada prueba, } \\
\text { también cambian pruebas y se } \\
\text { adiciona una prueba al HI del } \\
\text { OLTC }\end{array}$ \\
\hline 5 & 2013 & $\begin{array}{l}\text { J. Haema, R. } \\
\text { Phadungthin }\end{array}$ & $\% H I=\left(0.6 * \frac{\sum_{j=1}^{17}\left(K_{j} * H I F_{j}\right)}{\sum_{j=1}^{17} H I F_{j \max } * K_{j}}+0.4 * \frac{\sum_{j=18}^{21}\left(K_{j} * H I F_{j}\right)}{\sum_{j=18}^{21} 4 * K_{j}}\right) * 100$ & $\begin{array}{l}\text { Se trabaja con las mismas } 21 \\
\text { pruebas asignadas a la } \\
\text { metodología anterior, pero en } \\
\text { este caso cambia el método de } \\
\text { cálculo de la calidad del aceite }\end{array}$ \\
\hline 6 & 2014 & M. Augusta Martins & $H I=\left(X \% * \frac{\sum_{j=1}^{n}\left(K_{j} * S_{j}\right)}{\sum_{j=1}^{n} 4 K_{j}}+Y \% * \frac{\sum_{j=n+1}^{Z}\left(K_{j} * S_{j}\right)}{\sum_{j=n+1}^{Z} 4 K_{j}}\right) * 100$ & $\begin{array}{l}\text { Esta metodología trabaja con } 19 \\
\text { pruebas, } 3 \text { de ellas enfocadas en } \\
\text { el OLTC, pero a diferencia de las } \\
\text { demás se tiene en cuenta los } \\
\text { contactos del cambiador, los } \\
\text { pesos asignados a cada prueba } \\
\text { cambian todos. }\end{array}$ \\
\hline
\end{tabular}

Fuente: Adaptado de [18][19][20][21][22][23].

Con base en la investigación realizada, se decidió seleccionar 2 metodologías ítem 5 y 6 de el cuadro 1, basadas en técnicas de combinación lineal o pesos ponderados, para hacer la evaluación del subsistema del OLTC.

\section{Metodología para la evaluación de cambiadores de derivaciones bajo carga oltc sumergidos en aceite mineral}

Con base en las 2 metodologías mencionadas, se procedió con la construcción de la metodología para estimar el IS del OLTC. 
Pruebas para calcular el índice de salud del OLTC según la metodología planteada

\section{Análisis de gases disueltos (DGA) en el aceite del OLTC}

En el cuadro 2, se presenta el valor límite del factor de puntuación y ponderación para el DGA en el aceite del OLTC [22][23]. La puntuación se clasifica en seis niveles. El factor de puntuación y ponderación se calcula para obtener el factor DGA porcentual (\% DGATF) [23], para esto se utiliza la ecuación (1).

$$
\% D G A T F=\frac{\sum_{i=1}^{n} S_{i} * W_{i}}{\sum_{i=1}^{n}\left(S_{\max i} * W_{i}\right)} * 100
$$

Cuadro 2. Factor de puntuación y ponderación de DGA.

\begin{tabular}{|c|c|c|c|c|c|c|c|}
\hline \multirow{2}{*}{ GAS } & \multicolumn{6}{|c|}{ PUNTUACIÓN $\left(S_{i}\right)$} & $\begin{array}{r}W_{i} \\
(1-5)\end{array}$ \\
\cline { 2 - 6 } & 1 BUENO & 2 & 3 & 4 & 5 & 6 MALO & \\
\hline$\frac{C_{2}+H_{4}+C_{2} H_{6}+C_{2} H_{4}}{H_{2}+C_{2} H_{2}+C_{2} H_{6}}$ & $<0.5$ & N/A & N/A & N/A & N/A & $\geq 0.5$ & 4 \\
\hline$\frac{C_{4}+C_{2} H_{6}+C_{2} H_{4}}{C_{2} H_{2}}$ & $<2.0$ & N/A & N/A & N/A & N/A & $\geq 2.0$ & 4 \\
\hline$C_{2} H_{4}$ & $<1.0$ & N/A & N/A & N/A & N/A & $\geq 1.0$ & 4 \\
\hline$C_{2} H_{2}$ & & & & & & \\
\hline
\end{tabular}

Fuente: Adaptado de [22][23].

El HIF de \%DGATF resultante se muestra en el cuadro 3, dando así una clasificación de los resultados obtenidos del cálculo matemático, con la cual, se puede realizar una clasificación de la importancia en la intervención que deba tener este equipo bajo prueba.

Cuadro 3. Comparación HIF con \%DGATF para obtener una condición.

\begin{tabular}{|c|c|c|c|}
\hline Factor HI (HIF) & Condición & Descripción & Color \\
\hline 4 & Bueno & DGATF $\leq 25 \%$ & Verde \\
\hline 3 & Aceptable & $26 \% \leq$ DGATF $\leq 40 \%$ & Azul \\
\hline 2 & Regular & $41 \% \leq$ DGATF $\leq 60 \%$ & Amarillo \\
\hline 1 & Malo & $61 \% \leq$ DGATF $\leq 80 \%$ & Naranja \\
\hline 0 & Muy malo & DGATF $\geq 81 \%$ & Rojo \\
\hline
\end{tabular}

Fuente: Adaptado de [22][23]

\section{Calidad del aceite en el OLTC}

La evaluación de la calidad del aceite en el OLTC se realiza considerando tres pruebas de las presentadas a continuación, estas son: rigidez dieléctrica, contenido de agua y color, para cada prueba se tiene en cuenta el nivel de tensión de aplicación, en el cuadro 4, se puede observar que la separación de los GAP's en la prueba de rigidez dieléctrica es de $1 \mathrm{~mm}$, los resultados se clasifican con una puntuación entre 1 y 4 , siendo 1 bueno y 4 malo. 
Cuadro 4. Pruebas para la evaluación general de la calidad del aceite.

\begin{tabular}{|c|c|c|c|c|c|}
\hline \multirow[b]{2}{*}{ Pruebas } & \multicolumn{5}{|c|}{ Calidad del Aceite } \\
\hline & $U \leq 69 \mathrm{kV}$ & $\begin{array}{l}69 \mathrm{kV}<U< \\
230 \mathrm{kV}\end{array}$ & $U \geq 230 \mathrm{kV}$ & Puntuación & $W_{i}$ \\
\hline \multirow{4}{*}{$\begin{array}{l}\text { Rigidez dieléctrica } \\
\text { ASTM D1816 } \\
\text { Separación } 1 \mathrm{~mm}\end{array}$} & $>46$ & $>53$ & $>56$ & 1 = Bueno & \multirow{4}{*}{3} \\
\hline & $44-46$ & $51-53$ & 54-56 & 2 & \\
\hline & $42-44$ & $49-51$ & $52-54$ & 3 & \\
\hline & $<42$ & $<49$ & $<52$ & $4=$ Malo & \\
\hline \multirow{4}{*}{ IFT } & $\geq 32$ & $>40$ & $>40$ & 1 = Bueno & \multirow{4}{*}{2} \\
\hline & $27-32$ & $32-40$ & $32-40$ & 2 & \\
\hline & $22-27$ & $25-32$ & $25-32$ & 3 & \\
\hline & $\leq 22$ & $\leq 25$ & $\leq 25$ & $4=$ Malo & \\
\hline \multirow{4}{*}{ NN (Acidez) } & $\leq 0.05$ & $\leq 0.04$ & $\leq 0.03$ & 1 = Bueno & \multirow{4}{*}{1} \\
\hline & $0.05-0.1$ & $0.04-0.1$ & $0.03-0.07$ & 2 & \\
\hline & $0.1-0.2$ & $0.1-0.15$ & $0.07-0.1$ & 3 & \\
\hline & $\geq 0.2$ & $\geq 0.15$ & $\geq 0.1$ & $4=$ Malo & \\
\hline \multirow{4}{*}{ Contenido de agua } & $\leq 25$ & $\leq 15$ & $\leq 10$ & 1 = Bueno & \multirow{4}{*}{4} \\
\hline & $25-30$ & $15-20$ & $10-15$ & 2 & \\
\hline & $30-35$ & $20-25$ & $15-20$ & 3 & \\
\hline & $>35$ & $>25$ & $>20$ & $4=$ Malo & \\
\hline \multirow{3}{*}{ Color } & \multirow{3}{*}{\multicolumn{3}{|c|}{$\begin{array}{c}\leq 1.5 \\
1.5-2.0 \\
2.0-2.5 \\
\geq 2.5\end{array}$}} & 1 = Bueno & \multirow{3}{*}{2} \\
\hline & & & & 2 & \\
\hline & & & & $\begin{array}{c}3 \\
4-M-10\end{array}$ & \\
\hline
\end{tabular}

Fuente. Adaptado de [22].

El factor de puntuación y ponderación encontrado con los datos de la cuadro 4, son utilizados para calcular el factor de calidad del aceite porcentual (\% TOQF) usando la ecuación (2) y los límites se presentan a continuación en el cuadro 5.

$$
\% T O Q F=\frac{\sum_{i=R D}^{\text {Color }}\left(S_{i} * W_{i}\right)}{\sum_{i=R D}^{\text {Color }}\left(S_{\operatorname{maxi}} * W_{i}\right)} * 100
$$

Cuadro 5. Factor de calidad del aceite porcentual \%TOQF.

\begin{tabular}{|c|c|c|c|}
\hline Factor HI (HIF) & Condición & Descripción & Color \\
\hline 4 & Bueno & OQF/TOQF $\leq 25 \%$ & Verde \\
\hline 3 & Aceptable & $25 \%<\mathrm{OQF} / \mathrm{TOQF} \leq 40 \%$ & Azul \\
\hline 2 & Regular & $40 \%<\mathrm{OQF} / \mathrm{TOQF} \leq 60 \%$ & Amarillo \\
\hline 1 & Malo & $60 \%<\mathrm{OQF} / \mathrm{TOQF} \leq 80 \%$ & Naranja \\
\hline 0 & Muy malo & $\mathrm{OQF} / \mathrm{TOQF}>80 \%$ & Rojo \\
\hline
\end{tabular}

Fuente: Adaptado de [22].

\section{Inspecciones visuales para evaluar el OLTC.}

La inspección visual proporciona principalmente una evaluación externa semanal, mensual o anual. Se verifica el historial de carga, el escaneo térmico, la condición general externa y se examina el gabinete de control del OLTC, se necesita principalmente de la experiencia de un operador experto para dar un parte acertado de lo observado, por lo tanto, se sale del alcance de este trabajo. La mayoría de las fallas en cambiadores de derivación bajo carga se 
manifiestan generando calor. Por lo tanto, midiendo la diferencia de temperatura entre la cuba principal y el tanque del OLTC, es posible detectar defectos en el cambiador [15]. La gravedad del sobrecalentamiento definida para las termografías se califica de la siguiente manera, considerando el exceso de temperatura, ver cuadro 6.

Cuadro 6. Gravedad del sobrecalentamiento según la variación de temperatura.

\begin{tabular}{|c|c|}
\hline \multicolumn{2}{|c|}{ Gravedad del sobrecalentamiento } \\
\hline Condición & Variación de Temperatura \\
\hline Atención & $0-9^{\circ} \mathrm{C}$ \\
\hline Intermedio & $10-20^{\circ} \mathrm{C}$ \\
\hline Grave & $21-49^{\circ} \mathrm{C}$ \\
\hline Crítico & $>50^{\circ} \mathrm{C}$ \\
\hline
\end{tabular}

Fuente: Adaptado de [8][15].

\section{Evaluación de la condición de los contactos del OLTC}

La evaluación de los contactos se realiza según las recomendaciones de los fabricantes, teniendo en cuenta el material del que están compuestos los contactos, en esta metodología se evalúan los contactos de cobre, para los cuales la referencia de espesor es $25 \mathrm{~mm}$, siendo esta la medida estandarizada de fábrica y los desgastes mostrados en el cuadro 7 [24][25].

Cuadro 7. Recomendaciones para evaluar la condición de los contactos con un porcentaje de índice de salud.

\begin{tabular}{|c|c|c|c|}
\hline Condición observada & Descripción & Ĺmites [mm] & HI-CONTACTOS \\
\hline Sin desgaste & Sin deterioro observado & Desg $\leq 5$ & 1 \\
\hline Desgaste normal & $\begin{array}{c}\text { El componente activo es apto para un } \\
\text { servicio continuo. Hay poco deterioro }\end{array}$ & $5 \lessdot$ Desg $\leq 6.5$ & 2 \\
\hline Desgaste considerable & $\begin{array}{c}\text { Corrosión importante o des gaste } \\
\text { excesivo en componentes y cojinetes }\end{array}$ & $6.5 \varangle$ Desg $\leq 8$ & 3 \\
\hline Desgaste sustancial & $\begin{array}{c}\text { Corrosión importante o desgaste } \\
\text { excesivo en componentes y cojinetes }\end{array}$ & Desg $>8$ & 4 \\
\hline
\end{tabular}

Fuente: Adaptado de [24][25].

La evaluación de los contactos presenta un resultado final que varía entre 1 y 4 , siendo 1 la mejor condición y 4 un estado crítico, por lo que se decide trabajar con una razón de cambio encontrada utilizando la ecuación (3), para que el índice de salud tenga una salida entre 0 $100 \%$.

$$
H_{\text {CONT.F }[\%]}=\frac{100}{25}=4 \%
$$

Lo que indica que cada milímetro de cambio tendrá una variación de 4\% en el índice de salud, el resultado será porcentual y el OLTC recibirá una clasificación según el cuadro 8. 
Cuadro 8. Clasificación del OLTC según el estado de los contactos.

\begin{tabular}{|c|c|c|c|}
\hline \multicolumn{4}{|c|}{$\mathrm{HI}_{\text {Contactos }[\%]}$} \\
\hline Desgaste en $\mathrm{mm}$ & $\% H I$ & Condición & Clasificación HI \\
\hline 0 & 100 & \multirow{6}{*}{ Sin desgaste } & \multirow{6}{*}{1} \\
\hline 1 & 96 & & \\
\hline 2 & 92 & & \\
\hline 3 & 88 & & \\
\hline 4 & 84 & & \\
\hline 5 & 80 & & \\
\hline 6 & 76 & \multirow{2}{*}{ Seguimiento } & \multirow{2}{*}{2} \\
\hline 7 & 72 & & \\
\hline 8 & 68 & \multirow{2}{*}{$\begin{array}{c}\text { Desgaste } \\
\text { considerable }\end{array}$} & \multirow{2}{*}{3} \\
\hline 9 & 64 & & \\
\hline 10 & 60 & \multirow{2}{*}{$\begin{array}{c}\text { Desgaste } \\
\text { sustancial }\end{array}$} & \multirow{2}{*}{4} \\
\hline 11 & 56 & & \\
\hline
\end{tabular}

De las condiciones mostradas anteriormente, se puede decir que si los valores resultantes se encuentran dentro de los límites establecidos como rangos de operación la calificación será buena (1), regular (2) y mala (3) y muy mala (4).

\section{Cálculo del índice de salud del OLTC}

Finalmente, para el cálculo del índice de salud del OLTC se empleó un modelo matemático que realiza la combinación de los índices de salud individuales de cada prueba mencionada en lel cuadro 9, donde la combinación final de estos resultados será el índice de salud del OLTC.

$$
H I=\left(\frac{\sum_{j=n+1}^{Z}\left(K_{j} * S_{j}\right)}{\sum_{j=n+1}^{Z} 4 K_{j}}\right) * 100
$$

Donde $S_{j}$ : puntuación correspondiente al parámetro "j", $K_{\jmath}$ Factor de peso correspondiente al parámetro "j", j es el número de cada parámetro de diagnóstico y 4 el puntaje máximo, utilizado para el cálculo del Índice de salud del OLTC.

A continuación, en el cuadro 9, se presentan las pruebas utilizadas por esta metodología, la puntuación asignada a cada una dependiendo de los resultados y el peso que tendrá cada una en la evaluación final del índice de salud con la ecuación (4).

Cuadro 9. Pruebas de la metodología seleccionada, pesos y ponderaciones.

\begin{tabular}{|c|c|c|c|}
\hline \multicolumn{5}{|c|}{ Pruebas utilizadas para la evaluación del OLTC } \\
\hline \# de prueba & Parámetro para la condición del OLTC & $K_{j}$ & $S_{j}$ \\
\hline 1 & DGA en el aceite del OLTC & 6 & $4,3,2,1,0$ \\
\hline 2 & Calidad del aceite del OLTC & 3 & $4,3,2,1,0$ \\
\hline 3 & Gabinete de control del OLTC & 1 & $4,3,2,1,0$ \\
\hline 4 & Compartimiento del OLTC & 1 & $4,3,2,1,0$ \\
\hline 5 & Condición de los contactos del OLTC & 6 & $4,3,2,1,0$ \\
\hline
\end{tabular}


Para la clasificación final de la condición del OLTC por el índice de salud total HI TOTAL se emplearán los tres rangos mostrados en el cuadro 10.

Cuadro 10. Índice de salud total del OLTC.

\begin{tabular}{|c|c|}
\hline OLTC HItotal [\%] & Condición \\
\hline $\mathrm{HI}_{\mathrm{TOTAL}[\%]}>80$ & Bueno \\
\hline $60<\mathrm{HI}_{\mathrm{TOTAL}[\%]} \leq 80$ & Seguimiento \\
\hline $\mathrm{HI}_{\mathrm{TOTAL}[\%]} \leq 60$ & Crítico \\
\hline
\end{tabular}

Metodología utilizada por GRALTA para evaluar el transformador de potencia sumergido en aceite mineral.

La herramienta desarrollada por el grupo de investigación para evaluar el IS del transformador tiene una estructura por módulos, definidos por el tipo de prueba, que permite obtener índices de salud individuales, los cuales luego se combinan para obtener el índice de salud total ver figura 7 .

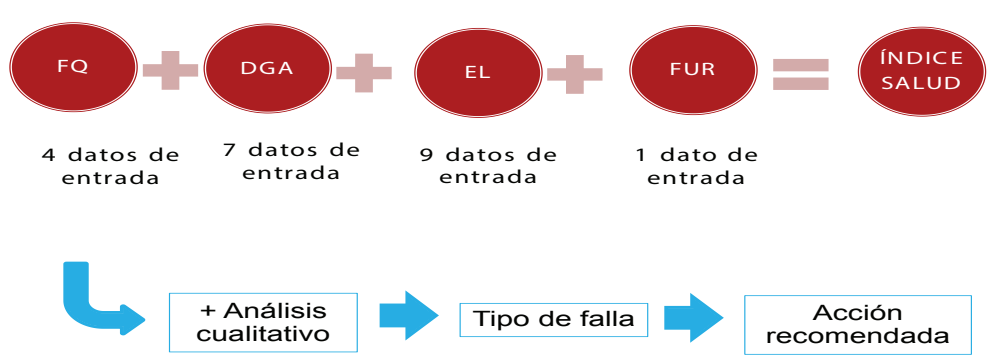

Figura 7. Estructura de la herramienta para la obtención del índice de salud de los transformadores. Fuente: Adaptado de Herramienta GRALTA

A cada prueba que se realiza se le debe dar un parámetro que califica los resultados obtenidos, luego a cada prueba o a su resultado (parámetro) se le asigna un peso y con la combinación de todos los parámetros y pesos, se obtiene el índice de salud, ver figura 8.

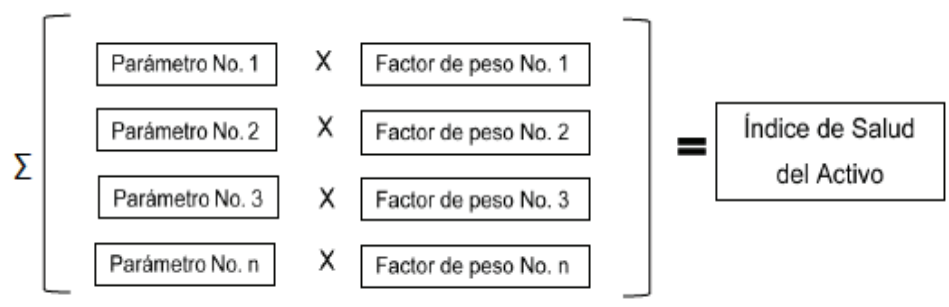

Figura 8. Proceso de combinación de parámetros. Fuente: Adaptado de Herramienta GRALTA 
Índice de salud del transformador de potencia considerando el subcomponente OLTC.

Se hizo la combinación de las 2 metodologías para obtener un índice de salud del transformador considerando el OLTC.

$$
H I=\left(X \% * \frac{\sum_{j=1}^{4} K_{j} * H I_{X}}{\sum_{j=1}^{4} 4 K_{j}}+Y \% * \frac{\sum_{j=1}^{4}\left(K_{j} * H I_{x}\right)}{\sum_{j=1}^{4} 4 K_{j}}\right) * 100
$$

Se asignó un porcentaje " $X$ " del $60 \%$ al índice de salud del transformador obtenido con la herramienta de GRALTA y un porcentaje "Y" del 40\% al índice de salud del OLTC, obtenido a partir de las pruebas implementadas en esta metodología. [21][18][19][20][22][23].

\section{Resultados}

Integración a la herramienta ya desarrollada por el grupo de investigación

la metodología desarrollada puede integrarse a la herramienta desarrollada por el grupo de investigación GRALTA, ya que:

- El método de combinación lineal utilizado en el cálculo del índice de salud en esta metodología, es el mismo que utiliza la herramienta desarrollada por GRALTA, donde la metodología propuesta tiene un porcentaje de influencia del $40 \%$ y la herramienta del grupo un 60\%, en la figura 9, se puede observar el planteamiento general para el cálculo del índice de salud considerando las pruebas que aportan información de la condición interna del equipo.

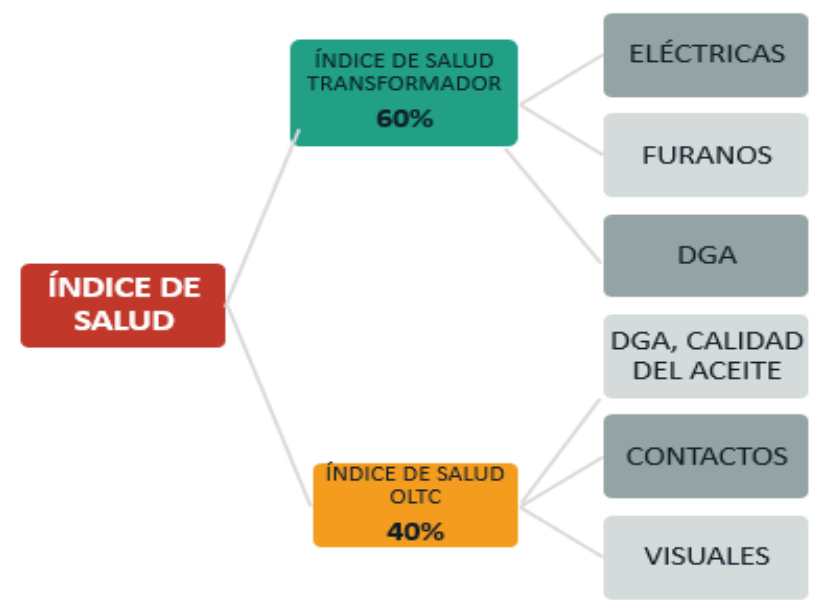

Figura 9. Asignación de pruebas para la obtención de índices de salud individuales y general.

- Ambas trabajan con el índice de salud como indicador de la condición final del transformador de potencia, basándose en las condiciones individuales de cada subsistema analizado.

- Al integrar el cálculo del índice de salud del OLTC, al cálculo de la condición del transformador, se mejora la estimación de la condición final, ya que se está analizando un subcomponente más de este activo ver figura 10. 


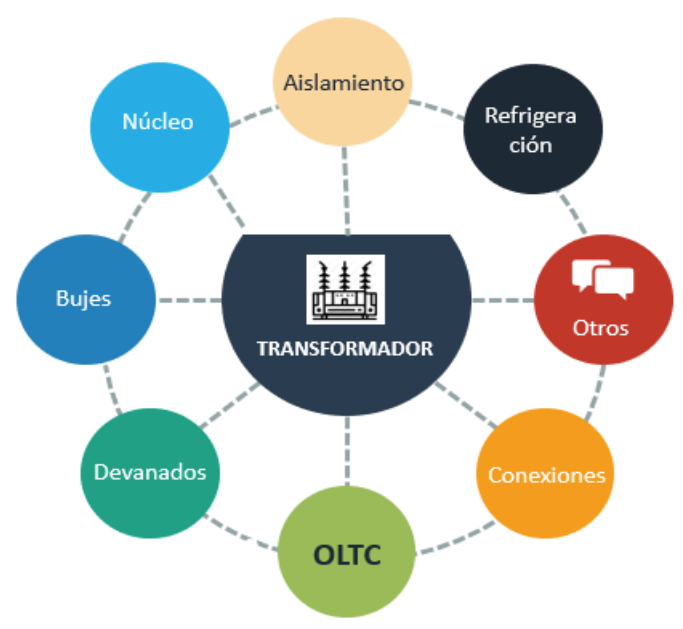

Figura 10. Subcomponentes del transformador de potencia. Fuente. Adaptado de [3] oil reclamation, testing methods for the determination of remaining insulation (paper.

\section{Validación de la metodología desarrollada en este trabajo}

Los datos presentados a continuación, hacen parte de un caso de estudio, el cual fue implementado como soporte para la validación de la metodología desarrollada en este trabajo, se trata de una de flota de 13 transformadores de potencia a los cuales se les nombrara desde T01 hasta T13, el tratamiento de los datos tiene el objetivo de calcular y analizar el índice de salud general de cada activo, para lo cual se debe aclarar que se contó con información de parte de la herramienta desarrollada por GRALTA.

El grupo de datos utilizado corresponde a mediciones entre los años 2017 y 2018, estos transformadores son de un nivel de tensión de $115 \mathrm{kV}$ y todos cuentan con OLTC debido a la importancia que tienen en el SEP para el cual operan. A continuación, se presentan los resultados de la evaluación.

Cuadro 11. Resultados del índice de salud total de los 13 transformadores evaluados.

\begin{tabular}{|c|c|c|c|c|c|c|}
\hline \multicolumn{1}{|c}{$60 \%$} & \multicolumn{2}{c|}{$100 \%$} \\
\hline SERIAL & $\begin{array}{c}\text { Health } \\
\text { Index trafo }\end{array}$ & Condición & $\begin{array}{c}\text { Índice de } \\
\text { salud del } \\
\text { OLTC }\end{array}$ & Condición & $\begin{array}{c}\text { Índice de } \\
\text { salud total }\end{array}$ & Condición \\
\hline T01 & 84.4 & Bueno & 95.3 & Bueno & 88.8 & Bueno \\
\hline T02 & 90.6 & Bueno & 73.8 & Seguimiento & 83.9 & Seguimiento \\
\hline T03 & 85.8 & Bueno & 87.0 & Bueno & 86.3 & Bueno \\
\hline T04 & 88.8 & Bueno & 65.7 & Seguimiento & 79.6 & Seguimiento \\
\hline T05 & 84.8 & Bueno & 87.5 & Bueno & 85.9 & Bueno \\
\hline T06 & 53.9 & Crítico & 58.3 & Crítico & 55.7 & Crítico \\
\hline T07 & 82.3 & Bueno & 75.1 & Seguimiento & 79.4 & Seguimiento \\
\hline T08 & 73.8 & Seguimiento & 56.5 & Crítico & 66.9 & Crítico \\
\hline T09 & 81.5 & Bueno & 83.8 & Bueno & 82.4 & Seguimiento \\
\hline T10 & 85.4 & Bueno & 94.7 & Bueno & 89.1 & Bueno \\
\hline T11 & 89.8 & Bueno & 77.3 & Seguimiento & 84.8 & Seguimiento \\
\hline T12 & 74.8 & Seguimiento & 93.9 & Bueno & 82.4 & Seguimiento \\
\hline T13 & 80.3 & Bueno & 56.2 & Crítico & 70.7 & Seguimiento \\
\hline
\end{tabular}




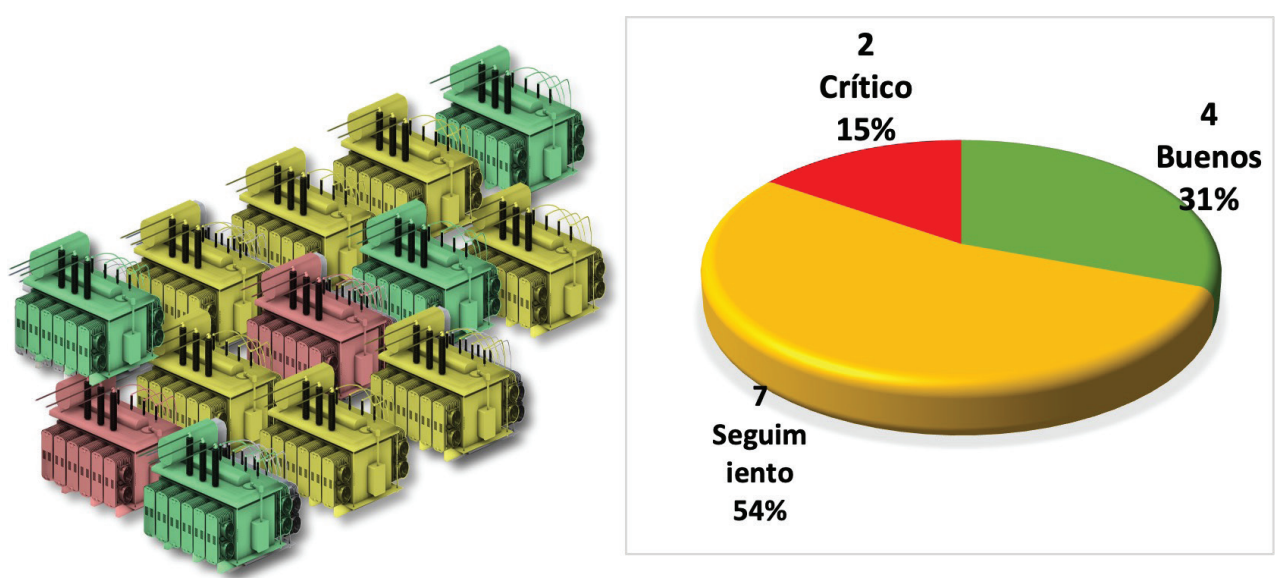

Figura 12. Índice de salud total del transformador de potencia incluyendo el OLTC.

\section{Conclusiones}

A través de la información analizada y recopilada en este trabajo, se logró confirmar que el OLTC es un elemento clave para el SEP, por lo tanto, se debe considerar en la estimación de la condición del transformador de potencia.

La metodología desarrollada en este trabajo permite hacer la evaluación del OLTC según su índice de salud, trabajando con pruebas que aportan información de la condición del equipo y tiene compatibilidad con la herramienta desarrollada en el grupo de investigación GRALTA, por lo que puede emplearse en empresas del sector eléctrico colombiano y de otros países de la región.

Los programas de gestión de activos en las empresas del sector se hacen cada vez más importantes, ya que estos conllevan a tener una mayor rentabilidad financiera en cuanto a planes de inversión, mejoras en la calidad y continuidad del servicio de energía eléctrica, mejoras en la eficiencia de los activos y extensión de la vida útil de estos. Siendo estos los mayores beneficios que se obtienen con estos programas, las empresas deben implementar sistemas que les permita adquirir y procesar los datos, generando así, información para la gestión del transformador, activo clave en la operación del sistema eléctrico de potencia.

\section{Referencias}

[1] A. F. A. Cerón, I. F. Orduña, G. Aponte, and A. A. Romero, "Panorama de la Gestión de Activos para Transformadores de Potencia Overview of Asset Management for Power Transformers," vol. 26, pp. 99-110, 2015, doi: 10.4067/S0718-07642015000300014.

[2] E. Chaidee and W. Tippachon, "Failure statistics and condition evaluation for power transformer maintenance," Asia-Pacific Power Energy Eng. Conf. APPEEC, pp. 1-4, 2011, doi: 10.1109/APPEEC.2011.5749108.

[3] IEEE Std C57.140, IEEE Guide for the Evaluation and Reconditioning of Liquid Immersed Power Transformers, no. April. 2017.

[4] OFGEM, Dno common network asset indices methodology, no. January. 2017.

[5] P. Picher et al., "New technologies for monitoring transformer tap-changers and bushings and their integration into a modern IT infrastructure," 44th Int. Conf. Large High Volt. Electr. Syst. 2012, no. August 2015, 2012.

[6] R. Frotscher., "Tap-changer know-how," Mag. Transform., vol. 3, no. 4, p. 24, 2013.

[7] J. J. Erbrink et al., "On-load tap changer diagnosis - An off-line method for detecting degradation and defects: Part 1," IEEE Electr. Insul. Mag., vol. 26, no. 5, pp. 49-59, 2010, doi: 10.1109/MEl.2010.5585008.

[8] R. Rodríguez and A. Yolima, Evaluación de la salud de activos de los transformadores de potencia de las subestaciones del área metropolitana del Valle de Aburrá. 2018. 
[9] M. Foata and C. Rajotte, "ON-LINE TESTING OF ON-LOAD TAP CHANGERS WITH A PORTABLE ACOUSTIC SYSTEM Raw data," vol. 1, pp. 293-298, 2000.

[10] P. Guillermo and A. Mayor, "PRUEBAS A CAMBIADORES DE TAPS EN TRANSFORMADORES DE Índice general," 2015.

[11] A. Boricic et al., "Dynamic resistance measurements and result interpretation for various on-load tap changers," 2019 IEEE Milan PowerTech, PowerTech 2019, pp. 1-6, 2019, doi: 10.1109/PTC.2019.8810530.

[12] C. Ranga, A. K. Chandel, and R. Chandel, "Condition assessment of power transformers based on multiattributes using fuzzy logic," IET Sci. Meas. Technol., vol. 11, no. 8, pp. 983-990, 2017, doi: 10.1049/ietsmt.2016.0497.

[13] A. Chantola, M. Sharma, and A. Saini, "Integrated Fuzzy Logic Approach for Calculation of Health Index of Power Transformer," Proc. Int. Conf. Inven. Commun. Comput. Technol. ICICCT 2018, no. Icicct, pp. 10451050, 2018, doi: 10.1109/ICICCT.2018.8473316.

[14] D. Dohnal, "On-load tap changers for power transformers," MR Knowl. Base, p. 24, 2013.

[15] S. Marcos et al., "' Estudio de transformadores con cambiador de derivaciones bajo carga ," pp. 1-8, 2015.

[16] D. J. Rogers and T. C. Green, "An active-shunt diverter for onload tap changers," IEEE Trans. Power Deliv., vol. 28, no. 2, pp. 649-657, 2013, doi: 10.1109/TPWRD.2013.2243171.

[17] L. R. Lewand and Paul Griffi, "Condition Assessment of Oil Circuit Breakers and Load Tap-Changers by the Use of Laboratory Testing and Diagnostics," pp. 1-4, 2004.

[18] A. Naderian, S. Cress, R. Piercy, F. Wang, and J. Service, "An approach to determine the health index of power transformers," Conf. Rec. IEEE Int. Symp. Electr. Insul., pp. 192-196, 2008, doi: 10.1109/ELINSL.2008.4570308.

[19] A. N. Jahromi, R. Piercy, S. Cress, J. R. R. Service, and W. Fan, "An approach to power transformer asset management using health index," IEEE Electr. Insul. Mag., vol. 25, no. 2, pp. 20-34, 2009, doi: 10.1109/ MEl.2009.4802595.

[20] G. Tanasescu, O. Dragomir, L. Voinescu, B. Gorgan, and P. V Notingher, "CIGRÉ Regional South-East European Conference Paper 110 Assessment of Power Transformers Conditions Based on Health Index Simtech International SRL Politehnica of Bucharest 3 HIDROELECTRICA SA - SH Bistrita Romania 1 SC," no. Rseec, pp. 1-8, 2012.

[21] J. Haema and R. Phadungthin, "Condition assessment of the health index for power transformer," Proc. - Power Eng. Autom. Conf. PEAM 2012, pp. 2-5, 2012, doi: 10.1109/PEAM.2012.6612413.

[22] J. Haema and R. Phadungthin, "Development of condition evaluation for power transformer maintenance," Int. Conf. Power Eng. Energy Electr. Drives, no. May, pp. 620-623, 2013, doi: 10.1109/PowerEng.2013.6635680.

[23] M. A. Martins, "Condition and risk assessment of power transformers: A general approach to calculate a Health Index," Cienc. e Tecnol. dos Mater., vol. 26, no. 1, pp. 9-16, 2014, doi: 10.1016/j.ctmat.2014.09.002.

[24] M. Reinhausen and T. D. Falkensteinstrasse 8, "Technical Data - General Section," 2010. www.reinhausen. com.

[25] M. Reinhausen, "Cambiador de tomas en carga OILTAP® MS," vol. 1, p. 38, 2010. 